\title{
Local characterisation of fluid flow in sandstone with localised deformation features through fast neutron imaging
}

\author{
S.A. Hall ${ }^{1}$, D.Hughes ${ }^{2}$ and S. Rowe ${ }^{2}$ \\ ${ }^{1}$ Laboratoire 3S-R, CNRS / Grenoble Universities, Grenoble, France \\ ${ }^{2}$ Institut Laue-Langevin, Grenoble, France
}

\begin{abstract}
Understanding fluid flow through rocks is of key interest in hydrocarbon production and $\mathrm{CO}_{2}$ sequestration, amongst other applications. Such fluid injection or extraction from subsurface reservoirs can be significantly modified (increased or decreased) by deformation and in particular by localised deformation features (fractures, shear bands and compaction bands). How such deformation alters fluid flow is however not well characterised experimentally. Measurement of fluid-flow distributions throughout a specimen requires techniques that can, first, see inside a test specimen and, second, see the fluid distinctly from the solid part. Therefore, neutron absorption imaging is well adapted to fluid flow monitoring in rocks as water is largely opaque to neutrons (i.e., it is highly absorbing) and rocks are generally less absorbing. In this paper we present initial results of neutron radiography monitoring of fluid-flow through samples of a sandstone containing localised deformation features (shear-bands). A comparison of flow through an intact specimen and flow through samples containing localised deformation features is presented that provides insight into the effect of localised deformation on the flow properties.
\end{abstract}

\section{Introduction}

Understanding fluid flow through rocks is of key interest in hydrocarbon production and $\mathrm{CO}_{2}$ sequestration, amongst other applications. Such fluid injection or extraction from subsurface reservoirs can be significantly modified (increased or decreased) by deformation and in particular by localised deformation features (fractures, shear bands and compaction bands). How such deformation alters fluid flow is however not well characterised experimentally. Standard laboratory approaches involve measuring flow from one end of a test specimen to the other, which will give some average measure over the whole specimen, i.e., the combined effect of flow through the (relatively-undeformed) matrix and the (locally) deformed part. To develop better simulation models of fluid flow in such deformed systems ideally requires an understanding of how the flow has been modified in the deformed part alone. It is thus of significant interest to measure the fluid-flow locally throughout a test specimen, including within the localised deformation features.

Measurement of fluid-flow distributions throughout a specimen requires techniques that can, first, see inside a test specimen and, second, see the fluid distinctly from the solid part. Obvious candidates for such analysis are x-ray methods (radiography, for a 2D view, and tomography, for a

This is an Open Access article distributed under the terms of the Creative Commons Attribution-Noncommercial License 3.0, which permits unrestricted use, distribution, and reproduction in any noncommercial medium, provided the original work is properly cited. 
$3 \mathrm{D}$ view) as has been previously demonstrated, including through localised deformation features (e.g., David et al., 2009). However, x-rays are sensitive primarily to density changes (the absorption of x-rays varies in a roughly linear sense with atomic number). Therefore, there needs to be significant density change, i.e., significant contrast between the porosity before and after fluid enters, to detect the passage of the fluid, which is often not the case. Therefore a propagating fluid front may not standout from the background in an x-ray image or will only do so when there is a significant saturation change. An alternative method is neutron imaging, as has been demonstrated by Dierick et al. (2005) and Cnudd et al. (2008), for example. Neutron imaging is similar in concept to x-ray imaging except that the absorption is quite "random" as a function of atomic number. Thus, with neutrons, it is possible to distinguish different isotopes of the same element and whilst water is largely opaque to neutrons (i.e., it is highly absorbing), heavy water is quite transparent. Furthermore, rocks are generally less absorbing than water. In fact the key benefit for fluid flow monitoring in rocks is that neutrons are extremely sensitive to the presence of hydrogen, which exists in many of the fluids that are of interest (water, oil...). Therefore, neutron imaging would seem to be particularly adapted to fluid flow monitoring in rocks.

This paper presents initial results of neutron radiography monitoring of fluid-flow through samples of a sandstone containing localised deformation features (shear-bands). A comparison of flow through an intact specimen and flow through samples containing localised deformation features is presented to provide insight into the effect of localised deformation on the flow properties.

\section{Experimental Method}

The experiments presented herein involved neutron radiography imaging (carried out at the Neutrograph instrument at the Institute Laue-Langevin (ILL) in Grenoble) to follow water imbibition into specimens of a sandstone. The flow-experiment set-up (see Figure 1) was relatively simple, involving a small reservoir in which the sample sits on elevated "feet". This reservoir was filled up with water to the bottom of the sample and the water passed into the sample by imbibition. The reservoir was filled through a pipe from outside the experimental area to allow neutron imaging, and thus monitoring of the fluid movement in the sample, from the first instance (the experimental area had to be fully sealed before opening the neutron beam). Neutron radiography images were captured on a high-speed (optical digital) camera using a scintillator to convert the transmitted neutron radiation into visible light (see Dierick et al. (2005) and Van Overberghe (2006) for more details). The resultant radiography images represent a $2 \mathrm{D}$ picture of the absorption of the neutrons through the specimen. Images were acquired in the initial stages of the tests at 5-15 second intervals, which allowed a good time resolution of the advancing fluid-front; later in the tests, as the flow slowed, the intervals were longer.

The rock being studied here is a sandstone from the Vosges mountains in France that is made up of $93 \%$ quartz, $5 \%$ microcline $1 \%$ kaolinite and $1 \%$ micas with an average porosity of about $22 \%$ and mean grain diameter of $300 \mu \mathrm{m}$ (see Bésuelle et al., 2000, for more details on this rock). The cylindrical specimens used in this case were cored perpendicular to bedding and have diameter and height of $40 \mathrm{~mm}$. One of the specimens was intact, i.e., was "as is" after coring and the two others had been previously deformed under triaxial compression at 20 and $50 \mathrm{MPa}$ confining pressures, respectively (these samples come from the experimental campaign of Bésuelle, 1999). The first of the deformed specimens developed a single, slightly-"open" shear band during loading and was only really held together in the central part of the specimen. The other specimen appeared, from the outer surface, to have a principle and a second minor shear-band, both of which can be considered quite "closed". The two localised specimens had a slightly reduced permeability at the lower surface (the face in contact with the water) due to the triaxial loading test conditions, but as the flow is seen to be dominated by the localised deformation features this is not a critical issue. 


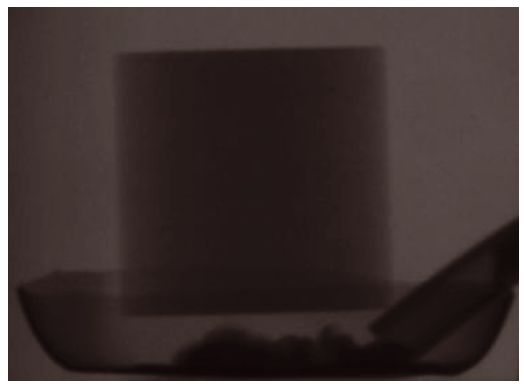

Figure 1 Experimental set-up with the sandstone core (40 $\mathrm{mm}$ diameter and $40 \mathrm{~mm}$ height) supported by three aluminium feet (not visible in this neutron radiograph image due to the low absorption of aluminium) implanted in "blutac" in the reservoir. The reservoir was a cut-off plastic container. The plastic pipe through which the water was injected into the reservoir can be seen to the right; the waterfront in the pipe can just be seen at the edge of the image

\section{Results}

Figures 2(a)-(c) show the neutron radiography images for water imbibition into the three study samples ((a) intact, (b) "open" shear-band and (c) "closed" shear-band). These images show the absorption of the neutron beam through the samples (and experimental set-up), dark colours indicating greater absorption. Water shows up as very dark since it has high absorption, the sample is grey and the open parts of the shear-bands appear lighter. It should be noted that for all the images presented very little image treatment was necessary - the water stands out quite clearly in the images and well above the noise level. The only treatment that has been carried out is outlier-removal to remove the very-low and very-high pixel values that correspond to bad pixels on the imager and to some noise probably associated with secondary gamma radiation.

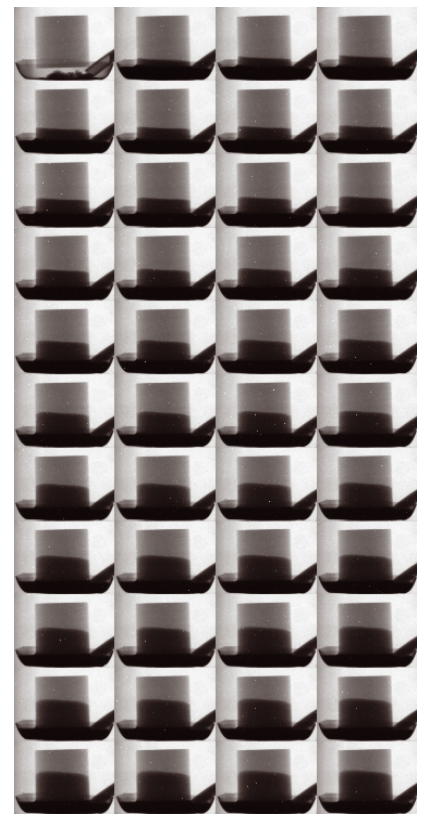

(a)

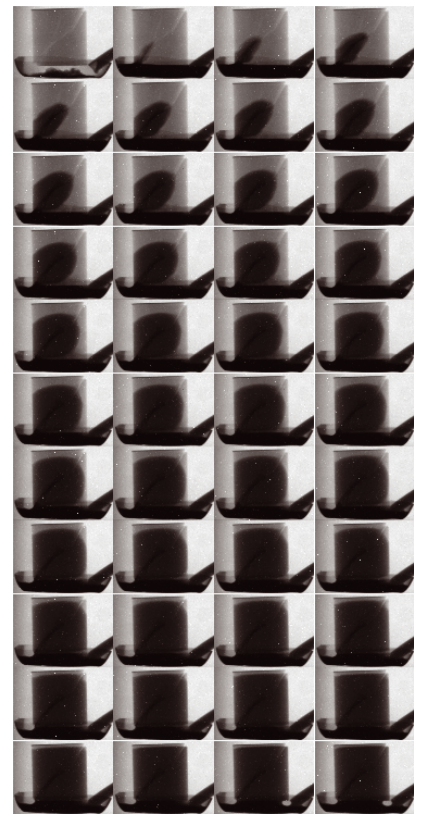

(b)

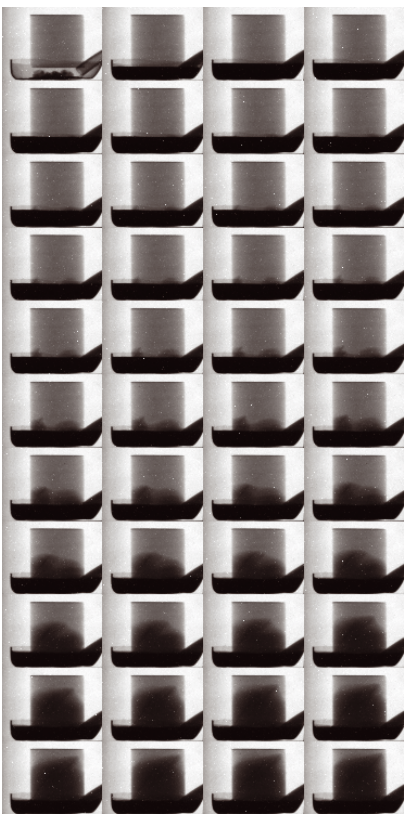

(c)

Figure 2 Time-lapse neutron radiography images of fluid imbibition into three Vosges sandstone samples :(a) intact specimen; (b) specimen deformed under $20 \mathrm{MPa}$ confinement; (c) specimen deformed under $50 \mathrm{MPa}$ confinement. The time intervals between each image are approximately 1015 seconds in each case (although the exact time does vary). 


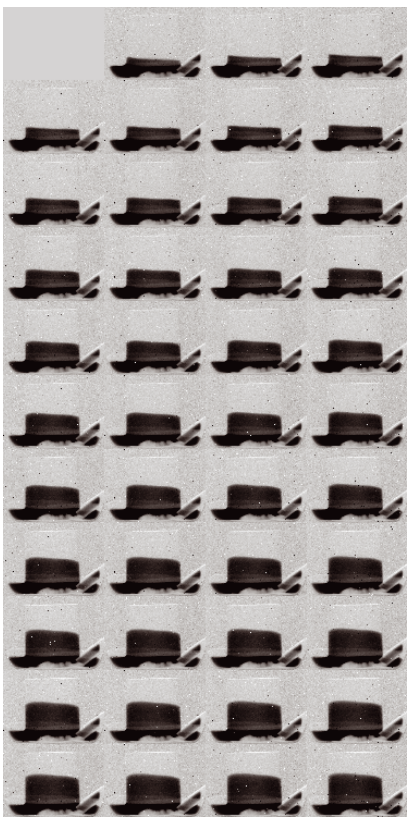

(a)

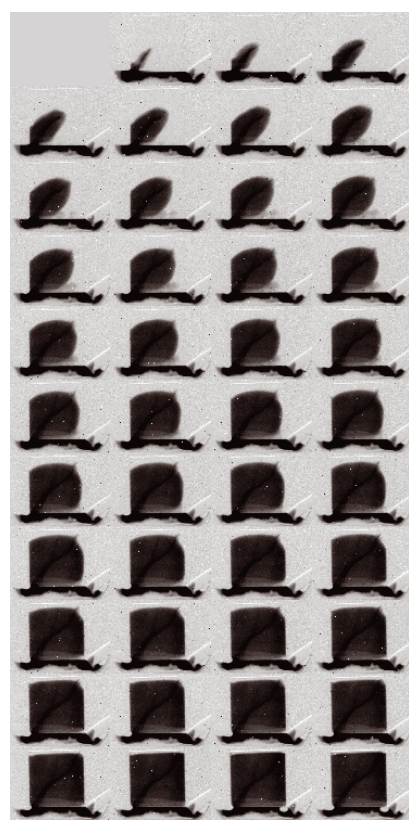

(b)

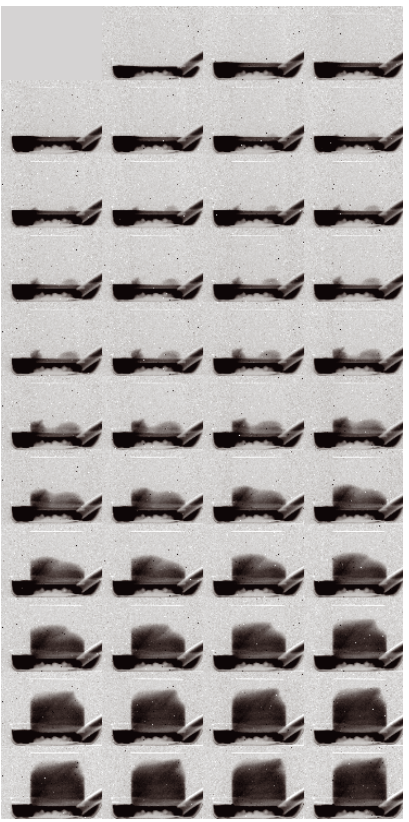

(c)

Figure 3 As Figure 2 but after subtraction of the first image of each sequence, which reveals a more representative image of the water saturation through the samples, for example the much higher saturation within the localised deformation features.

Some basic image manipulation can be carried out to reveal some more features of the flow and saturation distribution; Figure 3 shows the same images as in Figure 2 after subtraction of the first image of each sequence. This subtraction has the effect of removing the absorption due to the experimental set-up and the rock to show clearly the change in the image, i.e., the change in saturation. Thus this image can be considered to qualitatively represent a map of the water saturation in the sample, assuming zero saturation at the start. In particular these images show clearly that the localised deformation features have higher water saturation than the matrix.

In the time-lapse radiography images in Figures 2(a) and 3(a) the advancing water can be seen as a simple front ascending the specimen. This front is only deformed by the interaction with the sample boundary, which causes a retardation of the flow. The front is also slightly inclined, which is probably due to the sample not being vertical and perhaps also due to some non-horizontal fabric in the material. The flow-rate appears to vary with time only due to the distance of the front from the reservoir and there are no observable variations in flow properties in the sample.

For the specimen containing an "open" shear-band (Figures 2(b) and 3(b)) the water is seen to almost instantaneously enter the shear-band, after which it advances rapidly up the fracture. Diffusion from the fracture into the surrounding rock is seen at the same time. The front in the fracture advances ahead of the front in the matrix indicating that the propagation of the fluid within the fracture is faster than the diffusion into the matrix. This indicates that the flow is faster in the fracture, which might indicate higher permeability although, as this is capillary-driven flow, the flow velocity is a function of the pore-throat size as well as the permeability.

For the third specimen, with the "closed" shear-band (Figures 2(c) and 3(c)), the water advances much less rapidly than for the previous two specimens, both in the localised deformation zone and in the matrix, but the flow is seen to advance faster in the shear-band than outwith. Again diffusion of water from the fracture to the matrix is observed. 


\section{Next steps}

\section{Quantification of flow processes}

Clearly the results presented above provide direct insight into the modifications in the fluid flow due to the presence of the localised deformation. However to fully exploit such results requires that the flow observations be analysed in a quantified manner. The first step in this procedure is to identify and track the fluid-flow front in time and space, which can be used, for example, to calculate the variations in the fluid flow velocity in the deformed zones versus in the intact rock. Initial results from such an analysis are shown in Figure 3 (for a similar test, but for a different sample, to those present above). In these images the fluid-fronts have been picked at different (consecutive) stages of the flow by an automatic procedure using Akaike's Information Criterion in a similar approach to that commonly employed in seismology for picking seismic wave arrivals in seismograms. The fronts are picked from the radiograph images after despiking (high and low) and 2D smoothing (using a moving window mean filter). The result of the automatic picking can be seen from the example images to be quite successful. However problems arrive when the front becomes concave, i.e., beneath the more rapidly advancing part in the shear band. Work is underway to make the approach more general and thus capture these more complex features.

The flow direction vectors in Figure 3 have been defined from the local gradient of the fluid front at each stage, based on the assumption that the direction of the saturation profile decrease is the direction of flow. Using these directions to join up the picked flow fronts and finding the distance along them as a function of time between each step should thus give the local velocity of the flow in the sample.
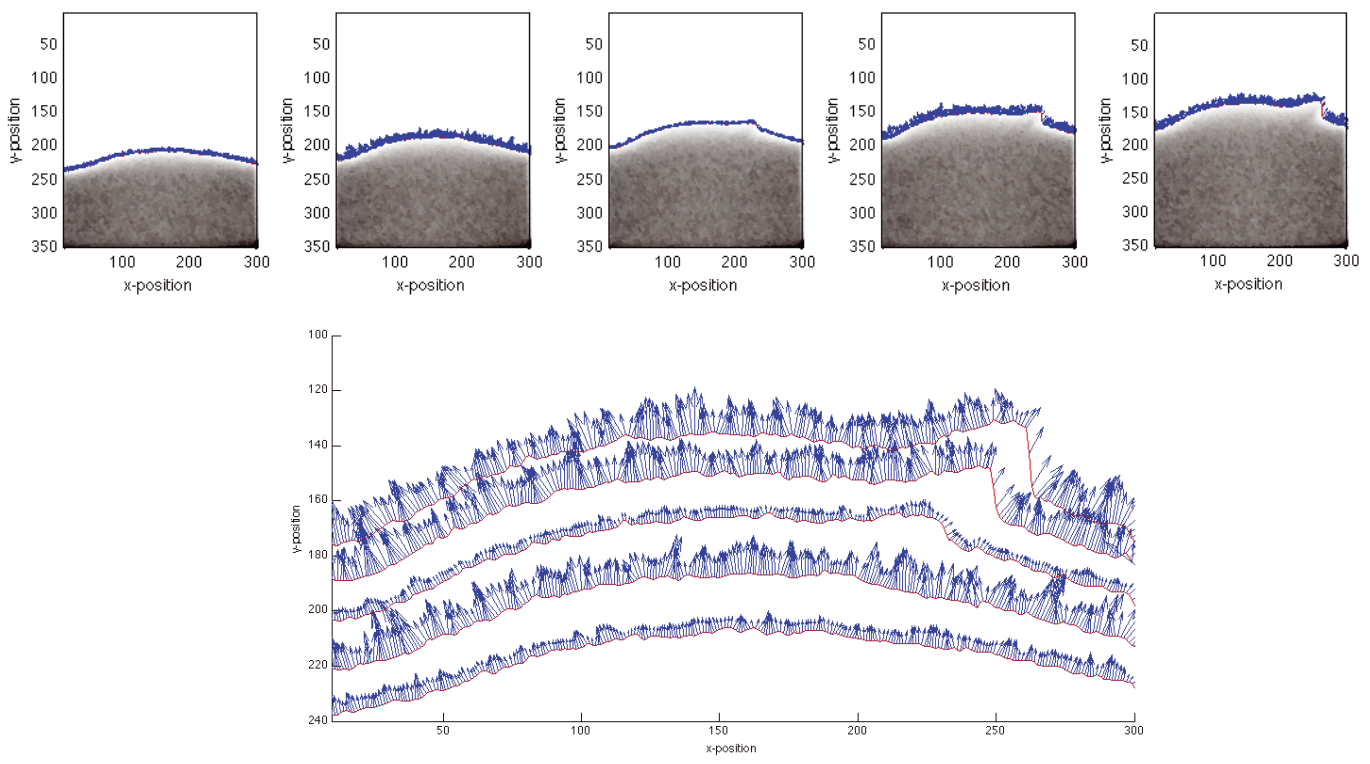

Fig. 3 Radiography images for water imbibition into a sample with a shear-band at 5 (approximately equal) time intervals plus automatically picked fluid fronts (red lines) and directions of maximum gradients of saturation at the front (blue vectors) - picked data are summarised in the lower image. 


\section{$3 D$ imaging of flow}

Figure 4 again shows radiography images taken during water imbibition into a sandstone sample with localised deformation, but in this case the sample was being rotated with the idea of acquiring $3 \mathrm{D}$ images. In the early stages of the imbibition the advance of the front was too fast relative to the rotation speed $(\approx 100 \mathrm{~s}$ for a full rotation) such that it was not possible to reconstruct the tomograms from the resultant images. However, these images provide, already, insight into the $3 \mathrm{D}$ aspect of the fluid-flow such that the fluid is seen to rise more rapidly up the edges of the sample and in particular up through the localised zone at the edges. Whilst some of this may be a surface effect on the flow, it is the opposite of that seen in the intact specimen above, where without any damage localisation the flow seems fastest up through the centre of the specimen. It is more likely that the effect is related to boundary effects in the mechanical loading, which led to the outer parts of the shear-band being more open than the regions nearer the centre of the sample. Thus the fluid travels faster up the shearband at the edges where it is more open (consistent with the open shear-band case above) and then diffuses into the matrix faster at the edge, as there is more fluid in the fracture there. Work is underway to improve the speed of acquisition (previously, using a more optimised set-up of this beamline full tomography scans were carried out in about $1 \mathrm{~s}$ - Van Overberghe, 2006). Towards the end of the imbibition, when the fluid has almost reached the top of the specimen and the flow velocity is relatively slow, it was possible to acquire a full 3D image, which showed that the fluid was homogeneously distributed in the rock mass outside of the shear band and the saturation the shear-band was also uniform, however the saturation was higher in the shear-band than in the matrix.

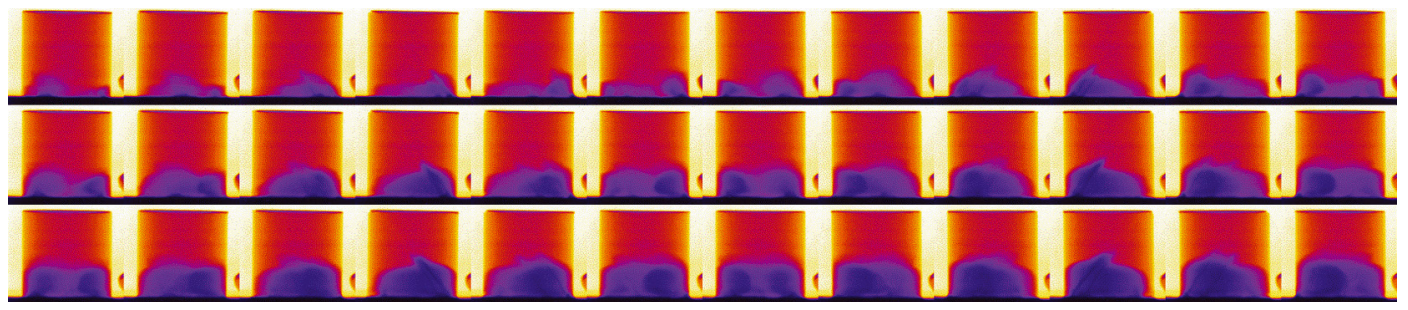

Fig. 4 Selected radiography images from 3 full rotations of a sample with a shear-band during water imbibition (1 rotation $\approx 100 \mathrm{~s}$, interval between images shown $\approx 8 \mathrm{~s}$ [actual acquisition interval was $\approx 0.4 \mathrm{~s}]$ ).

\section{Conclusions}

Initial results of neutron imaging to follow fluid flow through deformed rock specimens have been presented. The key aspect of the work is the observation of flow throughout a specimen, which provides the possibility to assess the local modifications to the flow due to (localised) deformation. These initial results show very clearly the flow processes in these sandstone samples and, more importantly, the modification of flow by localised deformation. In this case it appears that the shearbands represent conduits of increased water flow velocity, relative to the surrounding rock, in particular for the sample with a more open localised deformation zone. Also the localised deformation zones seem to have higher storage potential as they have higher water saturation. Further developments are underway to extract quantitative information on the flow properties such as local flow velocities and thus permeabilities. Initial 3D images indicate that $3 \mathrm{D}$ imaging is necessary and that the flow appears faster in the shear-band near the edges of the sample, probably due to a more dilatant behaviour during the deformation that created the shear-band feature. 


\section{Acknowledgements}

Andrew Harrison (Director of Science of the ILL) is gratefully acknowledged for his support of this work and for providing access to the Neutrograph facility at the ILL.

\section{References}

1. Bésuelle P., PhD thesis, University of Grenoble I, France (1999).

2. Bésuelle P., Desrues J. and Raynaud. S.. Int. J. Rock Mech. Mining Sci., 37, 8, 1223-1237 (2000).

3. Cnudde, V., Dierick, M., Vlassenbroeck, J., Masschaele, B., Lehmann, E., Jacobs, P., and Van Hoorebeke L., Nuclear Instruments and Methods in Physics Research B, 266,155-163 (2008).

4. David, C., Menendez, B., and Mengus, J.-M., Geophysical Research Letters, 35, L16313 (2009).

5. Dierick, M., Vlassenbroeck, J., Masschaele, B., Cnudde, V., Van Hoorebeke, L. and Hillenbach, A., Nuclear Instruments and Methods in Physics Research A, 542, 296-301 (2005).

6. Van Overberghe, A., PhD thesis, University of Heidelberg, Germany (2006). 